\title{
INTEGRATION OF BUILDING KNOWLEDGE INTO BINARY SPACE PARTITIONING FOR THE RECONSTRUCTION OF REGULARIZED BUILDING MODELS
}

\author{
Andreas Wichmann ${ }^{\mathrm{a}}$, Jaewook Jung ${ }^{\mathrm{b}}$, Gunho Sohn ${ }^{\mathrm{b}}$, Martin Kada ${ }^{\mathrm{a}}$, Manfred Ehlers ${ }^{\mathrm{a}}$ \\ ${ }^{a}$ Institute for Geoinformatics and Remote Sensing (IGF), University of Osnabrück, \\ Barbarastr. 22b, 49076 Osnabrück, Germany \\ firstname.lastname@uni-osnabrueck.de \\ ${ }^{\mathrm{b}}$ Department of Earth, Space Science and Engineering, York University, \\ 4700 Keele Street, Toronto, Ontario, Canada M3J 1P3 \\ (jwjung, gsohn)@yorku.ca
}

Commission III, WG III/4

KEY WORDS: Reconstruction, Three-dimensional, Building, Knowledge Base, Regularization, Point Cloud

\begin{abstract}
:
Recent approaches for the automatic reconstruction of 3D building models from airborne point cloud data integrate prior knowledge of roof shapes with the intention to improve the regularization of the resulting models without lessening the flexibility to generate all real-world occurring roof shapes. In this paper, we present a method to integrate building knowledge into the data-driven approach that uses binary space partitioning (BSP) for modeling the 3D building geometry. A retrospective regularization of polygons that emerge from the BSP tree is not without difficulty because it has to deal with the 2D BSP subdivision itself and the plane definitions of the resulting partition regions to ensure topological correctness. This is aggravated by the use of hyperplanes during the binary subdivision that often splits planar roof regions into several parts that are stored in different subtrees of the BSP tree. We therefore introduce the use of hyperpolylines in the generation of the BSP tree to avoid unnecessary spatial subdivisions, so that the spatial integrity of planar roof regions is better maintained. The hyperpolylines are shown to result from basic building roof knowledge that is extracted based on roof topology graphs. An adjustment of the underlying point segments ensures that the positions of the extracted hyperpolylines result in regularized 2D partitions as well as topologically correct 3D building models. The validity and limitations of the approach are demonstrated on real-world examples.
\end{abstract}

\section{INTRODUCTION}

Over the last two decades, 3D city and landscape models have significantly matured and their benefits for analyses, planning, and visualization purposes in the urban domain have since then been largely recognized. The most important objects of 3D city models are by far the buildings. In this paper, we are concerned in particular with building models with detailed roof structures and planar facades. Although such models can be manually or semi-automatically constructed, e.g., based on aerial images and $3 \mathrm{D}$ point clouds, the generation of large areas is only efficiently feasible by a fully automatic data interpretation. Even then, the reconstruction of buildings for larger cities can take up to many days. A still quite current overview on the topic of 3D building reconstruction is, e.g., given by Haala and Kada (2010).

Approaches for 3D building reconstruction are roughly divided into data driven and model driven approaches, depending on the degree of contextual knowledge they integrate about the general shape of buildings. Besides the assumption that almost all roofs consist of planar surfaces, pure data driven approaches do not integrate any other building knowledge at all. The shapes of the resulting 3D models are not limited by any restrictions and they resemble very closely the input data. Without regularization, however, the building models or parts thereof can easily end up distorted and exhibit small irregularities if the constructed planar surfaces do not precisely meet in common points or lines. Model driven approaches are more restricted towards the shapes that they are able to reconstruct. They often use a library of parameterized templates that can be combined to generate more complex shapes. The inherently strong regularization of shape templates is implicitly passed on to the reconstructed building models. Because not all buildings in the real world can be described by a set of shape templates, some buildings can only be crudely approximated by model driven approaches. Lately, data driven and model driven approaches have been merged towards hybrid reconstruction approaches that try to exploit the advantages of both worlds: the shape flexibility of a data driven approach with the regularization capabilities of a model driven approach.

The automatic 3D building reconstruction approach presented by Sohn et al. (2008) is in its core purely data driven. It uses the concept of binary space partitioning (BSP) to decompose the horizontal space according to the planar regions resulting from $3 \mathrm{D}$ point cloud segmentation. By assigning each resulting $2 \mathrm{D}$ region with its respective plane equation, a $3 \mathrm{D}$ building model is specified; see section 2 for specifics. The approach has been extended to also integrate general line regularization rules (Sohn et al. 2012) that improve the shape of surface boundaries, but maintain the plane information of the BSP regions. In this paper, we present a method to integrate explicit building roof knowledge in the form of roof shape types (saddleback, hip, asymmetric hip, etc.) to improve the generation of regularized BSP models and also to adjust the orientation and alignment of roof faces and roof elements. 


\section{BUILDING RECONSTRUCTION BASED ON BINARY SPACE PARTITIONING}

Binary Space Partitioning (BSP) is a hierarchical partitioning that recursively subdivides an $n$-dimensional space into convex subspaces by a set of (n-1)-dimensional hyperplanes. Based on an approach first utilized by Schumacker et al. (1969) it was originally developed in the context of 3D computer graphics for determining the visibility of surfaces during the rendering process of static scenes (Fuchs et al., 1980). Since then it has been adapted for a variety of applications such as ray-tracing (Naylor and Thibault, 1986), shadow generation (Chin and Feiner, 1989), solid modeling (Paterson and Yao, 1990), and image compression (Radha et al., 1996).

In the context of automatic building reconstruction a BSP based approach has been introduced by Sohn et al. (2008). It generates a polyhedral building model by applying the following three steps: point-wise clustering, building cue extraction, and BSP driven rooftop topology construction. The main aspects of each step are roughly summarized in the next paragraph.

The first step starts with a height clustering of all previously identified building points. It decomposes the initial set of points into clusters to reduce the shape complexity. Thereby, each cluster has the property that the height discrepancy between a point and its neighboring points, as defined by the Delaunay triangulation, is less than a predefined threshold. Then a plane clustering algorithm is independently applied to each height cluster in order to estimate segments. In the second step, intersection lines and step lines are extracted. The intersection lines are obtained for each height cluster by calculating the intersections of all pairs of adjacent segments in a height cluster. The extraction of the step lines is based on the boundaries of adjacent segments and a Compass Line Filter (CLF) (Sohn et al., 2008). For the rooftop topology reconstruction in the third step a $2 \mathrm{D}$ binary space partitioning is performed. Therefore, the extracted step lines and intersection lines from the previous step are formulated as hyperlines. Due to the recursive nature of the BSP, the resulting space partitioning depends on the order in which the hyperlines are applied. For this reason a partitioning score, which takes into account the plane homogeneity, the geometric regularity, and the edge correspondence for each hyperline, is calculated in every recursion. Finally, a merging process is performed based on the BSP tree that merges all those adjacent partitions whose planar equations have similar normal vectors.

This method is able to produce polyhedral building models even in complex urban settings where buildings are comprised of a number of sub-shapes. Additionally, it handles the presence of the missing data problem. However, due to the data-driven nature of this approach the quality of such a polyhedral model depends mainly on the extraction quality of the intersection and step lines. Even the devised geometric regularization of the CLF, which quantizes line slopes in a limited number of angular ranges, cannot always avoid irregular and sharp corners. Therefore, Sohn et al. (2012) introduce a method to rectify errors in a polyhedral building model. It considers the resulting vectors from a BSP as noisy model boundaries and progressively rectifies them based on a Minimum Description Length (MDL). The presented method is, on the one hand, able to produce building models which consider certain 2D regularizations. However, on the other hand, the solidness of the models is not guaranteed anymore, which means that a xy-coordinate can have more than one $z$-value due to unintentional gaps between adjacent roof planes. Some typical examples of this phenomenon are shown in Figure 1.
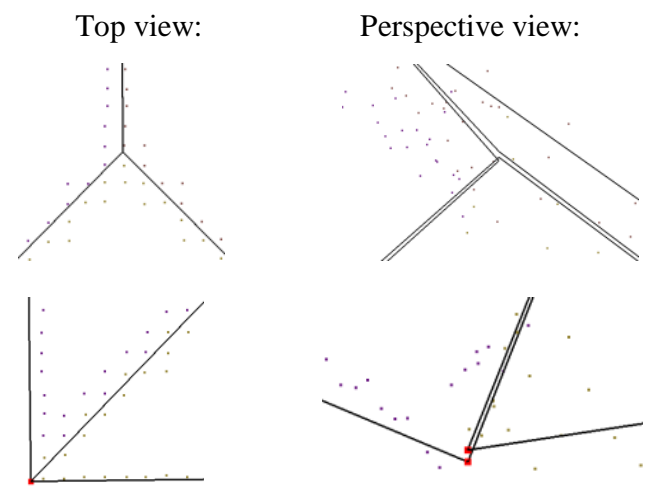

Figure 1. Two examples of unintentional gaps between roof planes after applying the MDL based optimization method presented in (Sohn et al., 2012).

A further optimization is presented in (Sohn et al., 2013). It takes additional hyperlines into account which are extracted from a single image. To connect these hyperlines with the already extracted hyperlines, different hypotheses based on CLF are generated and evaluated. An overview of the whole BSP based building reconstruction process is given in Figure 2 .

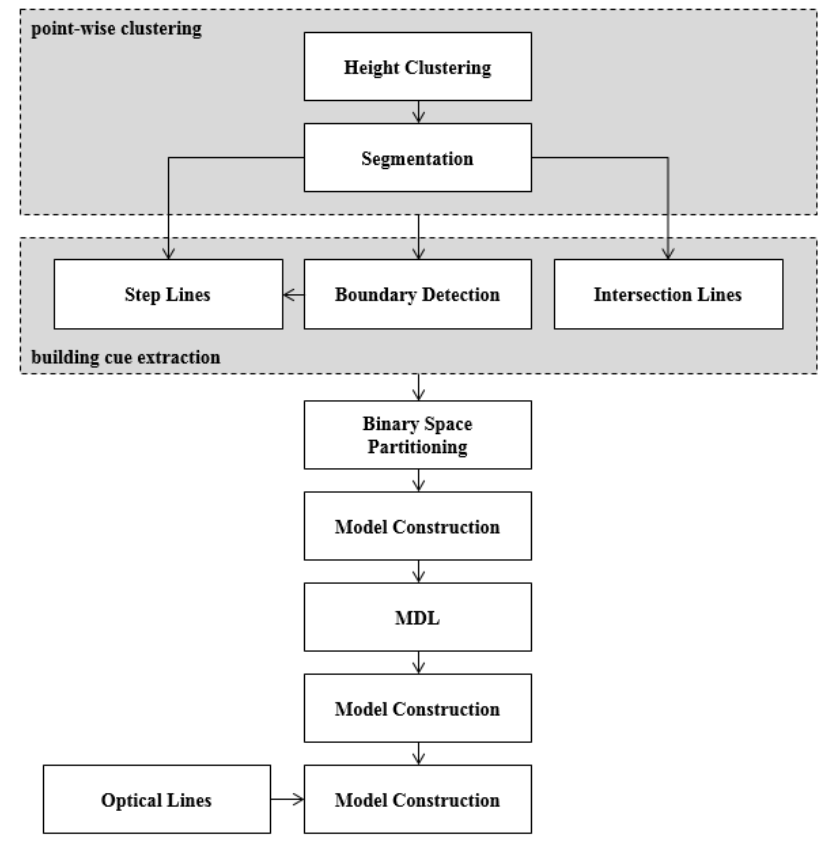

Figure 2. Overview of the reconstruction process based on BSP presented by Sohn et al. $(2008,2012,2013)$.

\section{RECONSTRUCTION PROCESS}

In this section, we first present our motivation and the central concept of our reconstruction approach. We explain in subsection 3.1 how building knowledge specifically can be used for the improvement of the resulting polyhedral model. Next, in subsection 3.2, we outline the workflow and the main components of our fully automatic reconstruction approach for regularized building models. 


\subsection{Motivation}

The BSP based building reconstruction approach described in the previous section is generally suitable for the reconstruction of planar building roofs. It handles the missing data problem and is not limited to certain roof types. The quality of such a polyhedral building model obtained by this approach, without applying any optimization method, depends mainly on the quality of the hyperlines, the sequence in which the hyperlines are applied (partitioning score), and the merging conditions for adjacent partitions. We believe that particularly these three aspects should be taken into account in a BSP based reconstruction process. Therefore, presented in this paper is a new fully automatic reconstruction approach which integrates building knowledge into binary space partitioning in order to

- improve the geometric accuracy of hyperlines,

- enhance the partitioning score,

- reduce the number of merge operations.

In order to achieve these objectives, we extract as much building knowledge as possible in a feature-driven process from certain parts of the point cloud. This knowledge is then used in different ways to support the subsequent data-driven reconstruction process. Thereby, the resulting polyhedral building model can be obtained directly from the BSP so that the construction of several polyhedral building models, which have to be optimized in an iterative way, is avoided. In addition, by integrating the building knowledge into the BSP we can guarantee that our regularized models are always closed. Furthermore, the ability to reconstruct the whole building and not just parts of it is not limited to the extractable building knowledge.

For this purpose, we also introduce in this paper the idea of hyperpolylines for the binary partitioning of the space in the context of building reconstruction. It reduces the number of partitions and supports the nonambiguity during the merging process of adjacent partitions. For example, the number of partitions for a hip roof without any superstructures can be reduced from six to four so that no further merging is needed. The reduction of the partition number has a big impact especially on buildings with small superstructures such as dormers which usually cause a large number of small partitions. Also, concave point sets can be directly expressed by the use of valid hyperpolylines. This allows a more natural partitioning of the space if such a partition can be detected directly from the point cloud.

A further advantage of the integration of building knowledge into BSP is that our method is able to define hyperlines in occluded building parts where otherwise no hyperlines can be extracted. Also, the merging criteria for two adjacent partitions are not limited anymore to the information of their own points. E.g. similar nonadjacent sub-parts of a building with the same semantic information can be taken into account.

\subsection{Workflow}

In this subsection we give an overview of the main parts of our reconstruction approach for the construction of regularized building models. It is based on a binary space partitioning which enables the integration of building knowledge. Thus, our reconstruction approach can generate a $3 \mathrm{D}$ polyhedral building model directly from a partitioned space so that natural regularities are considered and the final model is always solid and topologically correct. The process is presented in Figure 3 and consists of the following five main steps: point-wise clustering, building knowledge extraction, adjustments, building knowledge integration, and model construction.

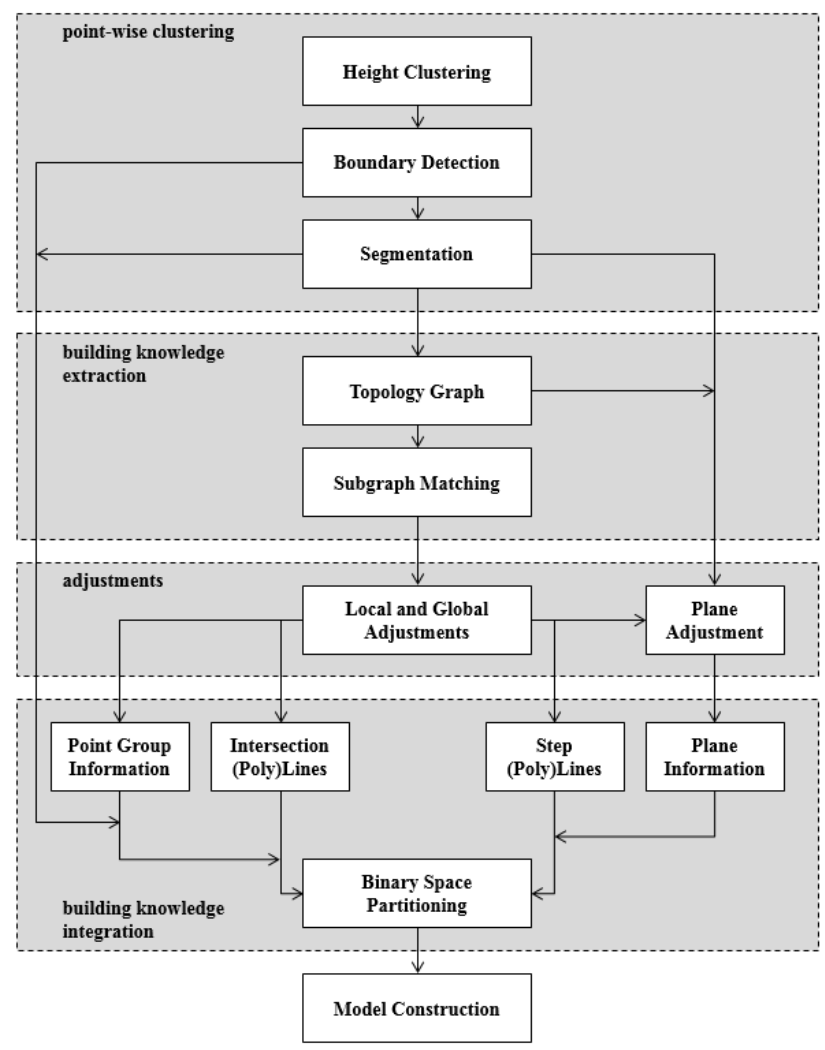

Figure 3. Overview of the extended BSP based reconstruction approach which integrates building knowledge for the construction of regularized models.

The workflow starts with a height clustering as described above. Based on the partitioning, we detect the $2 \mathrm{D}$ boundary for each height cluster. In cases where only one point set can be obtained the boundary represents the initial building outline. For the subsequent segmentation we perform sub-surface segmentation as introduced in (Kada and Wichmann, 2012) for each height cluster. Compared to common surface growing methods (see Vosselman and Klein, 2010), smaller segment patches, which are usually disconnected due to superstructures, are implicitly merged to larger segments so that the extraction of semantic information is improved.

Based on the result of the sub-surface segmentation, a roof topology graph (Verma et al., 2006) is constructed, where a node represents a segment and an edge between two nodes represents the relationship of two adjacent segments (see section 4). We use this topology graph to detect in a featuredriven recognition step certain properties and substructures of the building.

In the next step (see section 5), these properties and substructures are used for adjustment purposes. Thus, we first adjust those segments which are part of the extracted semantic information. After this all other segments which are not part of a recognized building feature are adjusted according to the 
properties of the extracted building knowledge. These two adjustment steps emphasize the natural structure in the geometry of a building so that a regularized space partitioning can be performed in the next step.

After the adjustments have been carried out, all relevant information is extracted for the construction of the BSP (see section 6). We provide point group information and adjusted intersection lines, step lines, polylines and plane information. Additionally, the original intersection lines, step lines and planes derived directly from the segmentation step, are also taken into account in not just the partitioning process of the space but also the order in which hyperlines and hyperpolylines are applied, and the merging process.

In the last step of the reconstruction process a regularized 3D polyhedral building model is directly obtained from the BSP.

\section{BUILDING KNWOLEDGE EXTRACTION}

For the automatic reconstruction of realistic 3D city models there currently exist several techniques that try to improve the shape of the constructed model with regard to the real world object. An effective method is the integration of building knowledge. We use the term building knowledge as a general term to summarize all information about a building. It includes inter alia the geometric, topologic, semantic, structural and regularity information of a building. The way it is used for reconstruction purposes is manifold.

Generally, building knowledge in data-driven approaches is commonly used for the reconstruction of small occluded parts or to emphasize the occurrence of symmetrical, parallel and orthogonal structures so that the geometric regularity in the resulting model can be perceptually enhanced. For these purposes, building knowledge is utilized to support repetitive structures and to define restrictions which avoid improbable and unnatural shapes. The restrictions affect for example the orientation of substructures or the slopes of roof planes. An example where building knowledge is taken into account for the reconstruction of building façades is given by $\mathrm{Pu}$ and Vosselman (2009). They first define several important building features such as walls, doors and windows based on the general knowledge of building façades for the recognition of these features in a segmented laser point cloud. Later, building knowledge is once again used to hypothesize the occluded building parts from the directly extracted features. Another example for the automatic construction of occluded building parts based on building knowledge is given by Becker (2009). She introduces a façade grammar which is automatically derived from successfully reconstructed parts of the building and applied to those parts which are occluded. An example which considers building knowledge to emphasize natural regularities in a building model is given in (Wichmann and Kada, 2014). In their fully automatic 3D building reconstruction approach they present several local and global regularization rules defined on planar half-spaces that integrate building knowledge to improve the shape and the accuracy of an automatic reconstructed building model.

In contrast to data-driven methods, the impact of building knowledge in purely model-driven approaches is usually greater. It is not only limited to the quality of the resulting model but also to the ability to reconstruct a building. Only building shapes of a predefined knowledge base can be reconstructed. Usually the knowledge base consists of several templates which represent different types of roofs. Once the best fitted template to the input data is detected, only an instance of it has to be estimated. These methods usually produce good results even for point clouds with low density. However, they are always strongly limited to the predefined building knowledge. Therefore, as more building knowledge in the form of templates is available, more different types of buildings can be reconstructed at the expense of computation time. Two recent examples for model-driven approaches are given in (Henn et al., 2013; Huang et al., 2013).

The building knowledge considered in our approach is also defined by several templates. However, in contrast to purely model-driven approaches the ability to reconstruct a building in our method is not limited to the predefined knowledge base. Analogous to data-driven approaches it affects only the quality of the resulting model.

For the extraction of building knowledge we adapted the roof topology graph presented in (Xiong et al., 2014). For each building a directed roof topology graph (dRTG) is created with $\mathrm{G}=\left(\mathrm{V}, \mathrm{E}, \mu_{\mathrm{v}}, \mu_{\mathrm{E}}, \mathrm{s}, \mathrm{t}\right)$, where $\mathrm{V}$ is the finite set of labeled vertices (nodes), $\mathrm{E} \subseteq\left\{\left(\mathrm{v}_{\mathrm{i}}, \mathrm{v}_{\mathrm{j}}\right) \mid \mathrm{v}_{\mathrm{i}}, \mathrm{v}_{\mathrm{j}} \in \mathrm{V}, \mathrm{v}_{\mathrm{i}} \neq \mathrm{v}_{\mathrm{j}}\right\}$ the finite set of labeled edges, $\mu \mathrm{v}$ the finite set of node attributes, $\mu \mathrm{E}$ the finite set of edge attributes, and the unary operations $\mathrm{s}$ and $\mathrm{t}$ with $\mathrm{s}: \mathrm{e} \rightarrow \mathrm{v}_{1}$ and $\mathrm{t}: \mathrm{e} \rightarrow \mathrm{v}_{2}$ for a given $\mathrm{e} \in \mathrm{E}$. A segment is represented in a dRTG as a labeled node, and the relationship between two adjacent segments as two directed labeled edges connecting the nodes with each other. We define that two segments are adjacent to each other if at least one point exists in each segment so that the distance between them is less than a predefined threshold. Thereby, we differentiate between twoand three-dimensional adjacency, ignoring the z-coordinates of the points in the two-dimensional distance calculation, to distinguish between potential step and intersection lines. This information is represented by an attribute of the edge. Additionally, we label each edge with a confidence value. For the calculation of the confidence value of a potential intersection line, we hypothesize between its two adjacent segments an intersection line that results from the intersection of those two planes which can be directly derived from the points of each segment. The confidence value is based on the following three criteria:

- the average distance of the segment points to their related segment planes,

- the number of segment points which are close to the hypothesized intersection line,

- the overlap length of the two segments with regard to their intersection line.

For the calculation of the overlap length, first all points close to the hypothesized intersection line are projected onto the line. Then, for each segment the longest line segment is estimated by using the outmost points on the intersection line that initially belong to the segment. The overlap length of both line segments yields the overlap length of the segments. The confidence value for potential step lines can be estimated in a similar way.

Based on the dRTG structure, we define a knowledge base with several subgraph templates for the recognition of typical building features. This includes for example ridge lines, valleys, dormers, and also different types of roof ends such as gable, hip, Dutch gable, bonnet and mansard. The occurrence of each 
template of the knowledge base is detected in the dRTG by performing an iterative subgraph matching algorithm which takes the node and edge labels into account. If an occurrence is found, it adds this semantic information as new attributes to the nodes and edges of the dRTG so that they can be used in the next iterations for the recognition of further building features. The recognition of building features stops if no further attribute can be added to the graph.

Afterwards, a second iterative recognition step is performed. It mainly considers the semantic information gained from the previous recognition step to identify simple substructures which often occur in a building. Thereby, those parts of the dRTG are preferred for the recognition where high confidence values are present. The confidence value of a building feature can automatically be modified during this recognition step if the dRTG gives hints for its correctness. For example, the confidence value of a dormer is improved if another dormer on the opposite site of an already detected mansard roof has been recognized. Additionally, the confidence value of a building feature is reduced if the same feature type has already been detected in the direct neighborhood. Thereby, a more or less balanced distribution of recognized substructures is accomplished in the dRTG. The recognition of substructures stops if the rate of segments, which are not part of any recognized substructure, is less than a predefined threshold or if no further substructures can be found in the graph. As mentioned before, the number of templates can be limited to reduce the computation time due to the fact that for reconstruction the whole building does not have to be represented by the predefined knowledge base.

\section{ADJUSTMENTS}

Based on the intrinsic information about a building gained from the dRTG in the previous step, the details of the adjustment that emphasizes regularities in the resulting model are explained in this section. The adjustment step is divided into two separate sub-steps. In the first sub-step, only those building parts that are represented by the dRTG are adjusted where building knowledge could be extracted. Then, in the second sub-step all other parts are adjusted according to the results of the first substep.

In analogy to the adjustment methods presented in (Wichmann and Kada, 2014), we differentiate between local and global adjustments. However, instead of half-spaces we apply our adjustment rules to segments and their plane equations. A local adjustment is performed on a set of adjacent segments that contribute to a roof component and which are therefore represented as a connected subgraph in the dRTG. Thus, local adjustments are individually applied for every connected subgraph and independent from other subgraphs. In the subsequent global adjustment special care is taken of the relation between the recognized subgraphs, even if they are not adjacent to each other. The two-step adjustment ensures that those segments which are part of a substructure have a higher impact on its segments than the segments of other substructures.

The adjustments in our approach change mainly the slope and the orientation of segments in a local and global context. By using a divisive clustering method, those segments which feature similar slopes or xy-directions are adjusted to their average value. Additionally, all segments of a substructure are translated within a strict predefined threshold during the global adjustment so that building features in the dRTG are merged together. In contrast to (Wichmann and Kada, 2014), during the local and global adjustment process, we also consider the semantic and the topological information of each segment. For example, we restrict the adjustment of the segments of a subgraph, which is surrounded by a segment of another subgraph, to remain in their respective segment. Otherwise the BSP tree would need to be altered; a process that can become quite complicated (dependent on the structure of the tree and the location of the segments within this tree). Therefore, dormers always remain on the same roof plane so that a possible degeneration of the building model is reduced. Furthermore, we ensure that the xy-direction of a subgraph, which represents a dormer, is mainly influenced by the main direction of its connected substructure. Because the number of roof shape templates is limited, the described adjustment process is quite efficient.

Subsequently, more divisive clustering algorithms are carried out in the second sub-step to adjust also those parts of the dRTG where no building knowledge could be extracted. For this purpose the global adjustments are repeated but this time including all segments and by considering the following three conditions:

- If more than one adjusted segment occurs in a cluster, the cluster is split again.

- If exactly one adjusted segment occurs in a cluster, all unadjusted segments are adapted to its value.

- If no adjusted segment occurs in a cluster, all segments are adjusted to their average value.

The combination of both adjustment steps exposes the natural structure in the geometry of a building so that a regularized space partitioning can be generated in the next step.

\section{BUILDING KNOWLEDGE INTEGRATION}

In this section, we explain how the extracted building knowledge is integrated into the BSP to produce regularized models. Without considering any building knowledge, the space partitioning can be performed based solely on the segmentation and boundary detection results, from which intersection and step lines are then formulated as hyperlines. The quality of such a partitioning is especially low for low density point clouds because the real orientations of the hyperlines are difficult to determine. Furthermore, this approach usually produces numerous small partitions as shown in Figure 4 which leads to ambiguities during the merging process.

For this reason, during the building knowledge integration step, we first collect all inner intersection and step lines that are part of a recognized substructure in the dRTG. Due to the adjustments in the previous step these lines are regularized and increase the occurrence of symmetrical, parallel and orthogonal structures. Afterwards, all building features which connect two substructures in the dRTG are used for the estimation of additional lines. Furthermore, also those parts of the building where no knowledge has been extracted are considered as follows: The segments are first categorized into different groups. Two groups are merged together if they are part of the same substructure or if they belong to two different groups which are connected by a building feature. Then, the intersection and step lines between two groups are added. Finally, the lines of the boundary detection are also added. 

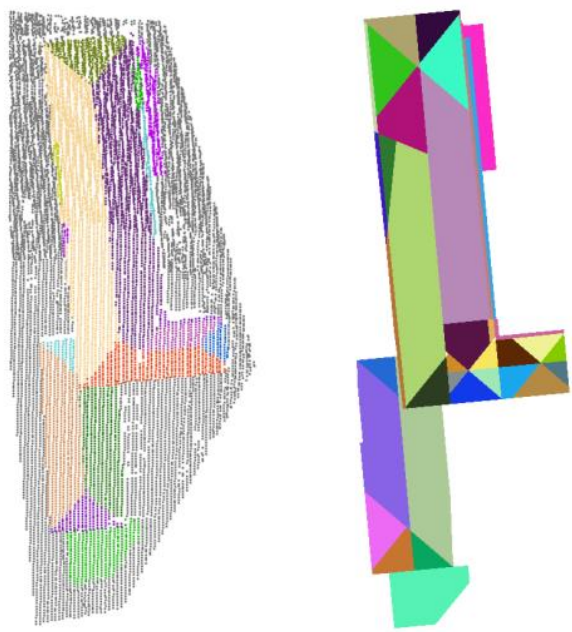

Figure 4. Left: The result of the segmentation. Right: The result of the binary space partitioning without considering building knowledge.

An example of the line generation can be found on the left side of Figure 5 where the input points that belong to the building are separated into four height clusters represented by different colors. The building knowledge extraction step detects a total of three one-sided hip roofs whose inner lines are colored in red. Two of them are in the same height cluster and a building feature in the dRTG indicates an L-connection relationship between them. Due to the different ridge line heights of the two connected one-sided hip roofs, the three green intersection lines are extracted based on the plane equations of the segments. Additional lines between two segments which belong to different groups are colored in blue. The black lines are derived from the boundary detection.
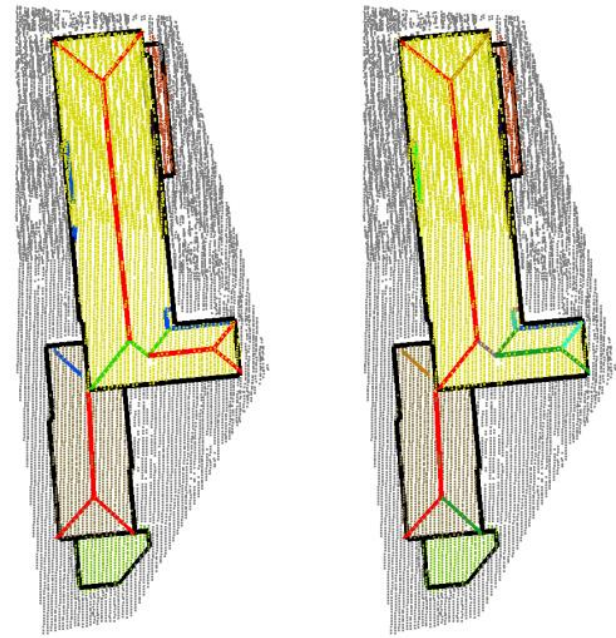

Figure 5. Left: Point cloud overlaid with extracted line segments originating from substructures (red), additional building knowledge (green) and segment groups (blue). Right: Point cloud overlaid with accumulated polylines colored for each height cluster according to their priority.

To reduce the number of partitions in the BSP tree, we treat all intersection and step lines as line segments and connect them to form polylines. The start and end points for line segments that are inside a recognized substructure are well defined through their shape templates. The two end points for other lines are determined as described in section 4. The polylines are estimated for each height cluster separately as follows: Choose the longest line segment that is not yet part of any polyline and repeatedly add the longest line segment (that is also not yet part of any polyline) that connects to either end point of the polyline to this new polyline until no more line segments can be added. Repeat until no more polylines can be generated in this way. The result of the polyline estimation is shown on the right side of Figure 5. The first extracted polyline of each height cluster in this figure is highlighted in red, the second in dark green, the third in brown, and so on. As shown, because of the polyline formulation as the partitioning element, the binary partitioning can now also be realized with concave borders which often occur in roof tops.

For the binary partitioning of a height cluster, all polylines are used and formulated as hyperpolylines. The order in which they are recursively applied has an impact on the result. Thus, we use a partitioning score which is calculated for each hyperpolyline and updated during every iteration of the partitioning process. It prioritizes hyperpolylines that are long, have a large number of line segments, and where the ratio of the polyline length and the length of the corresponding hyperline is close to 1 . As the points at the two sides of a partition should preferably be homogenous, the score also incorporates a factor that considers the number of points with similar planar properties in each partition. The result of the binary space partitioning with incorporated building knowledge is shown on the left side of Figure 6. Compared to the original partitioning in Figure 4, the number of partitions is now less than half.
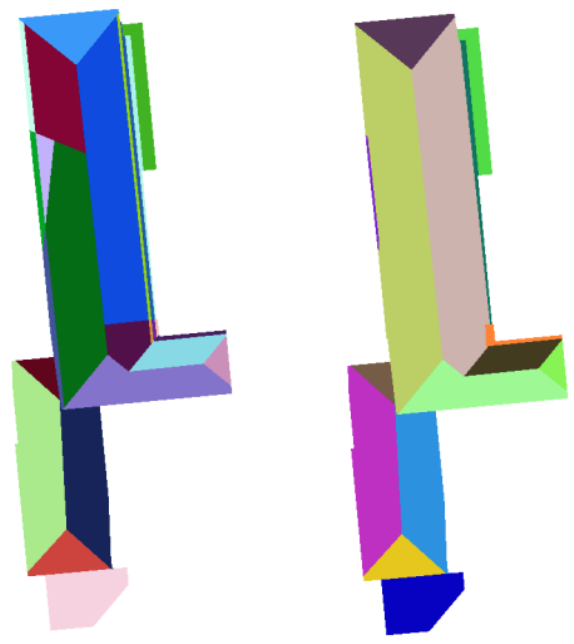

Figure 6. The result of the binary space partitioning which takes building knowledge into account before (left) and after (right) the merging process.

After space partitioning, adjacent partitions with similar normal vectors are merged and the plane equations of the new partitions are re-estimated. Due to the adjustment step the resulting plane equation of a partition depends not only on the points in it. Once all partitions with similar normal vectors are merged together, the outline of the regularized building can be extracted directly. During the conversion of the BSP tree into a boundary representation, the outline will result in façade polygons. 


\section{RESULTS}

We have tested our fully automatic building reconstruction approach on several selected buildings of the Vaihingen test data set $\left(4\right.$ points $/ \mathrm{m}^{2}$ ) provided by the German Society for Photogrammetry, Remote Sensing and Geoinformation (DGPF) (Cramer, 2010). In general, the approach presented in this paper is suitable for the reconstruction of buildings as already shown in the Figures of the previous section. It is not limited to the number of templates; it handles the missing data problem, and always produces closed and regularized building models which can be directly obtained from the BSP. For complex buildings with many sub- and superstructures in particular, we discovered a big improvement in the resulting polyhedral building model due to fewer partitions and the more regularized structure of the BSP. The quality of the model in our reconstruction approach depends mainly on the building knowledge extraction. The more knowledge that can be derived from the input data, the more regularized the BSP and its resulting model will be.
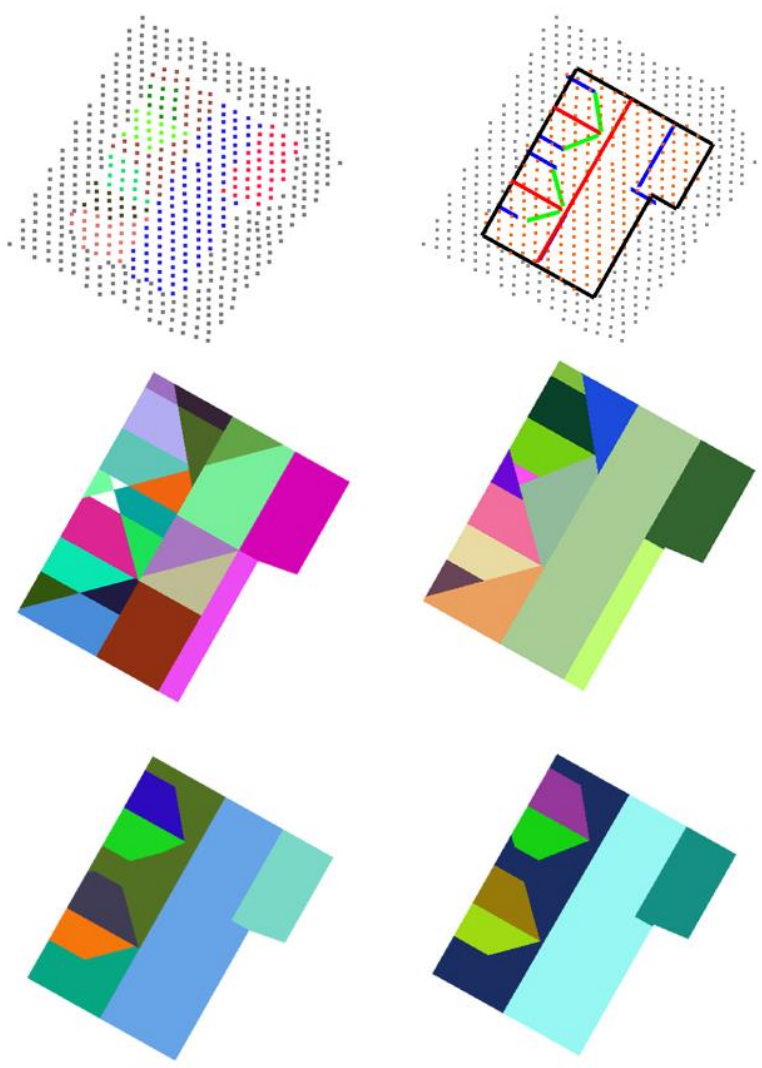

Figure 7. Left column: The result of the segmentation using a RANSAC plane extraction method and the BSP without considering building knowledge before and after the merging process. Right column: The result of the line extraction based on building knowledge and the building knowledge considering BSP before and after the merging process.

For test purposes, instead of the sub-surface segmentation, we also tried the RANSAC plane extraction method which was originally used in (Sohn et al., 2008). The result of the segmentation is presented in Figure 7. Based on this, we constructed the BSP once with (as shown in the right column) and once without (as shown in the left column) the integrated building knowledge in the BSP. As shown in the second row of
Figure 7, the number of partitions before applying the merging process is now reduced from 23 partitions to only 14 partitions. The main difference after the merging process is that the left roof plane on which the two dormers are located could not be merged together in the first case. This also has an impact on the quality of the bottom dormer because the black partition implies a longer ridge line than the orange one. Therefore, the resulting model of the BSP without considering any building knowledge has a gap. In contrast to this, the solidness of the reconstructed building model of our approach is shown in Figure 8.

An unsolved issue with our new approach which still occurs in the resulting outline is shown in Figure 8 and in its partitions in Figure 7. The dormer in the back misses a small part that is close to the building outline. It is incorporated in the roof plane because the hyperline from the other dormer cuts this part away. Similar problems also occur without the integration of building knowledge, as can be seen in the dark segment in the bottom left image of Figure 7, and is an unavoidable glitch in the greedy strategy of the partitioning process.
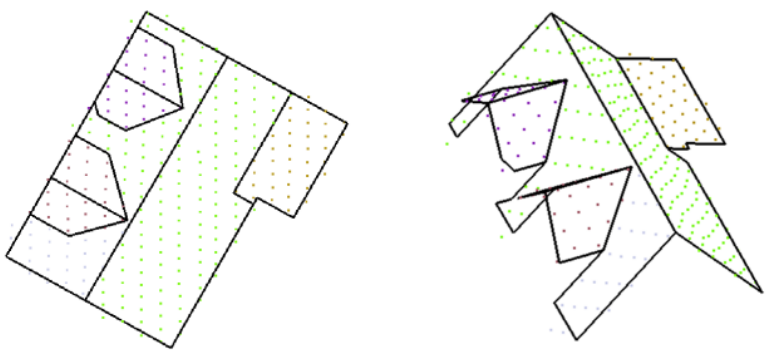

Figure 8. The resulting regularized building outline of our automatic reconstruction approach which is directly extracted from the BSP in top and perspective view.

The final result of our BSP based reconstruction approach for the two segmented point clouds in Figure 4 and Figure 7 is shown in Figure 9 once separately in magnified form, and once as part of the reconstructed test area 1 of the Vaihingen data set.
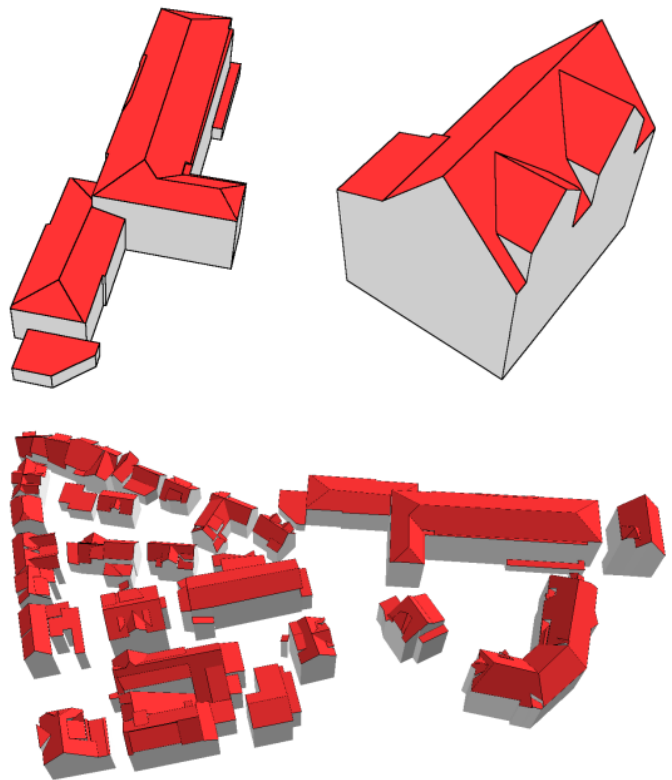

Figure 9. Resulting building models reconstructed by our BSP based reconstruction approach. 


\section{CONCLUSION}

In this paper, we presented a fully automatic building reconstruction approach that generates regularized models directly from the BSP. In contrast to already existing BSP based methods, the resulting solid building models feature a better geometric regularization. This is accomplished by extracting building knowledge in a feature-driven process and its integration into the BSP generation. Thereby, the BSP tree is enriched with model knowledge. To support the nonambiguity during the merging process of adjacent partitions we reduced the number of partitions inter alia by providing further point group information and the introduction of hyperpolylines in the BSP. The latter one enables the concave partitioning of a space so that the occurrence of smaller convex partitions is reduced. The presented approach is especially suitable for complex buildings with many sub- and superstructures. However, further investigations of the partitioning score are necessary so that a partitioning sequence can be accomplished that better maintains the integrity of the point cloud segments. Furthermore, a BSP framework should be developed with a strong focus on the tight integration of building knowledge, so that hyperpolylines can be directly hypothesized from the BSP itself.

\section{ACKNOWLEDGEMENTS}

The Vaihingen data set was provided by the German Society for Photogrammetry, Remote Sensing and Geoinformation (DGPF) (Cramer, 2010).

\section{REFERENCES}

Becker, S., 2009. Generation and application of rules for quality dependent façade reconstruction. In: ISPRS Journal of Photogrammetry and Remote Sensing, 64(6), pp. 640-653.

Chin, N., Feiner, S., 1989. Near real-time shadow generation using BSP trees. In: Computer Graphics, 23(3), pp. 99-106.

Cramer, M., 2010. The DGPF-test on digital aerial camera evaluation - overview and test design. In: Photogrammetrie Fernerkundung - Geoinformation, 2010(2), pp. 73-82.

Fuchs, H., Kedem, Z. M., Naylor, B. F., 1980. On visible surface generation by a priori tree structures. In: Computer Graphics, 14(3), pp. 124-133.

Haala, N., Kada, M., 2010. An update on automatic 3D building reconstruction. In: ISPRS Journal of Photogrammetry and Remote Sensing, 65(6), pp. 570-580.

Henn, A., Gröger, G., Stroh, V., Plümer, L., 2013. Model driven reconstruction of roofs from sparse LIDAR point clouds. In: ISPRS Journal of Photogrammetry and Remote Sensing, 76, pp. 17-29.

Huang, H., Brenner, C., Sester, M., 2013. A generative statistical approach to automatic 3D building roof reconstruction from laser scanning data. In: ISPRS Journal of Photogrammetry and Remote Sensing, 79, pp. 29-43.

Kada, M., Wichmann, A., 2012. Sub-surface growing and boundary generalization for 3D building reconstruction. In: The International Annals of Photogrammetry, Remote Sensing and Spatial Information Sciences, I-3, pp. 233-238.
Naylor, B. F., Thibault, W. C., 1986. Application of BSP trees to ray-tracing and CSG evaluation. Technical Report GIT-ICS $86 / 03$, Georgia Institute of Technology, School of Information and Computer Science.

Paterson, M. S., Yao, F. F., 1990. Efficient binary space partitions for hidden-surface removal and solid modeling. In: Discrete \& Computational Geometry, 5(1), pp. 485-503.

$\mathrm{Pu}$, S., Vosselman, G., 2009. Knowledge based reconstruction of building models from terrestrial laser scanning data. In: ISPRS Journal of Photogrammetry and Remote Sensing, 64(6), pp. 575-584.

Radha, H., Vetterli, M., Leonardi, R., 1996. Image compression using binary space partitioning trees. In: IEEE Transactions on image processing, 5(12), pp. 1610-1624.

Schumacker, R. A., Brand, B., Gilliland, M. G., Sharp, W. H., 1969. Study for applying computer-generated images to visual simulation. Report AFHRL-TR-69-14, U.S. Air Force Human Resources Laboratory.

Sohn, G., Huang, X., Tao, V., 2008. Using a binary space partitioning tree for reconstructing polyhedral building models from airborne LIDAR data. In: Photogrammetric Engineering and Remote Sensing, 74(11), pp. 1425-1438.

Sohn, G., Jwa, Y., Jung, J., Kim, H. B., 2012. An implicit regularization for $3 \mathrm{D}$ building rooftop modeling using airborne LIDAR data. In: The International Annals of Photogrammetry, Remote Sensing and Spatial Information Sciences, I-3, pp. 305310 .

Sohn, G., Jung, J., Jwa, Y., Armenakis, C., 2013. Sequential modelling of building rooftops by integrating airborne LIDAR data and optical imagery: preliminary results. In: The International Annals of Photogrammetry, Remote Sensing and Spatial Information Sciences, II-3/W1, pp. 27-33.

Verma, V., Kumar, R., Hsu, S., 2006. 3D building detection and modeling from aerial LIDAR data. In: Proceedings of the 2006 IEEE Computer Society Conference on Computer Vision and Pattern Recognition (CVPR'06). IEEE Computer Society, Washington, DC, pp. 2213-2220.

Vosselman, G., Klein, R., 2010. Visualisation and structuring of point clouds. In: Airborne and Terrestrial Laser Scanning, Vosselman, G., Maas, H.-G. (Eds.), Whittles Publishing, Scotland, UK, pp. 45-81.

Wichmann, A., Kada, M., 2014. 3D Building adjustment using planar half-space regularities. In: ISPRS Annals of the Photogrammetry, Remote Sensing and Spatial Information Sciences, II-3, pp. 189-196.

Xiong, B., Oude Elberink, S., Vosselman, G., 2014. A graph edit dictionary for correcting errors in roof topology graphs reconstructed from point clouds. In: ISPRS Journal of Photogrammetry and Remote Sensing, 93, pp. 227-242. 